\title{
Interprofessionelle Zusammenarbeit ist der Schlüssel
}

\section{Monika Bachmann}

Freie Journalistin

\author{
Sie vernachlässigen die Körperpflege oder ernähren sich mangelhaft. Und in ihrer \\ Wohnung steht es nicht zum Besten. Die Rede ist von älteren Menschen, die sich \\ selbst vernachlässigen. Die interprofessionelle Zusammenarbeit, insbesondere von \\ Ärzteschaft und Spitex, optimiert in solchen Fällen die Chance, den Betroffenen \\ nachhaltig zu helfen.
}

Bei der «Spitex RegionKöniz» geht die Meldung ein, dass Norbert K.* nach dem Austritt aus dem Spital Behandlungspflege benötigt. Der 85-Jährige wurde mit einer offenen Wunde am Bein in die Klinik eingewiesen. Der Patient ist mehrfachkrank: Die Ärzte diagnostizierten nebst dem Ulcus cruris auch eine Herz- und Niereninsuffizienz. Norbert K. ist mit der Spitex bereits vertraut: Seine Frau, die vor einigen Jahren verstarb, wurde vom ambulanten Pflegeteam betreut. Dennoch steht der alleinlebende Mann der ärztlich verordneten Hilfe durch die Spitex skeptisch gegenüber. Die Wunde muss zweimal pro Tag gepflegt werden. Und auch beim Duschen benötigt Norbert K. Unterstützung. Schon bald macht der Patient klar, dass er wieder für sich selbst sorgen will. Daran kann auch seine Tochter nichts ändern, die mit der Situation des kranken und betagten Vaters zunehmend an ihre Grenzen stösst. Allen involvierten Personen ist klar, dass sich Norbert K. mit seiner Haltung einer Selbstgefährdung aussetzt, denn die hygienischen Verhältnisse in seiner Wohnung sind alarmierend, die Körperpflege ist ungenügend und die Ernährung mangelhaft.

Selbstvernachlässigung im Alter

Wie gehen Ärztinnen und Ärzte sowie Pflegefachpersonen damit um, wenn Menschen, die dringend Hilfe benötigen, diese ablehnen? Und wie finden Fachleute den Zugang zu Personen, denen die Bewältigung des Alltags über den Kopf wächst und die deshalb Angst haben, die Kontrolle über ihr Leben zu verlieren? Diesen Fragen widmet sich die neue Publikation der Reihe «Praxiswissen» mit dem Titel "Selbstvernachlässigung im Alter - ein Thema der Gesundheitsförderung?», die vom Berner Bildungszentrum Pflege herausgegeben wird. Im ersten Teil geht es um die Bedeutung des Themas für die Gesellschaft und Pflege. Im zweiten Teil werden Zusammenhänge zwischen dem sich im Alter verändernden Selbstkonzept und dem Phänomen der Selbstvernachlässigung aufgezeigt. Im dritten Teil ergründen die Autorinnen, unter welchen Voraussetzungen betroffene Menschen Hilfe annehmen können, um ihre Gesundheit und damit ihre Unabhängigkeit zu schützen.

Carola Sroka, Sonja Bächler-Mäder. Selbstvernachlässigung im Alter - ein Thema der Gesundheitsförderung? Schriftenreihe Praxiswissen. Berner Bildungszentrum Pflege. Bern: hep-Verlag 2014. 48 Seiten. 19 CHF.

\section{Grosses Misstrauen}

«Dies ist ein typischer Fall von Selbstvernachlässigung im Alter», sagt Sandra Sermier, Fachleiterin Pflege bei der «Spitex RegionKöniz». «Der Patient ist multimorbid, weist ein Selbstversorgungsdefizit auf und äussert den Wunsch, zu Hause bleiben zu können.» Es handelt sich hierbei allerdings nicht um Personen mit einem klassischen Diogenes-Syndrom (umgangssprachlich «Messies» genannt), wie es angehende Ärztinnen und Ärzte im Medizinstudium kennenlernen, sondern um Menschen, die altersbedingt nicht mehr in der Lage sind, für sich selbst zu sorgen. Die Spitex wird immer wieder mit solchen Situationen konfrontiert. Und die demographische Entwicklung dürfte dem Thema zukünftig noch mehr Brisanz verleihen. Auch Ärztin Erika Eicher Vella, die in Neuenegg eine Praxis für Allgemeinmedizin betreibt, kommt regelmässig mit Menschen in Kontakt, die alleine nicht mehr zurechtkommen und trotzdem keine fremde Hilfe annehmen wollen. Häufig bekomme sie Hinweise von Angehörigen oder Nachbarn: «Sie sorgen sich um einen ihnen nahestehenden oder bekannten Menschen", so Erika Eicher Vella. Auch während des Notfalldienstes wird sie mit dem Thema konfrontiert: "Manchmal ruft mich jemand wegen einer Bagatelle an.» Zwischen den Zeilen höre sie dann heraus, dass das Problem anderswo liege. Sucht sie die Person auf, trifft sie nicht selten auf Zustände, die auf eine Selbstgefährdung hindeuten. In vielen Fällen kommt der Ärztin im Erstkontakt mit Betroffenen eines entgegen: "grosses Misstrauen.» Da Hausärztinnen und Hausärzte zunehmend zur «Mangelware» werden und ihr knappes Zeitbudget Hausbesuche nicht im grossen Stil zulässt, kann eine gute Zusammenarbeit und Kommunikation im Dreieck Patient-Hausärztin-Spitex für den Erfolg ausschlaggebend sein. 


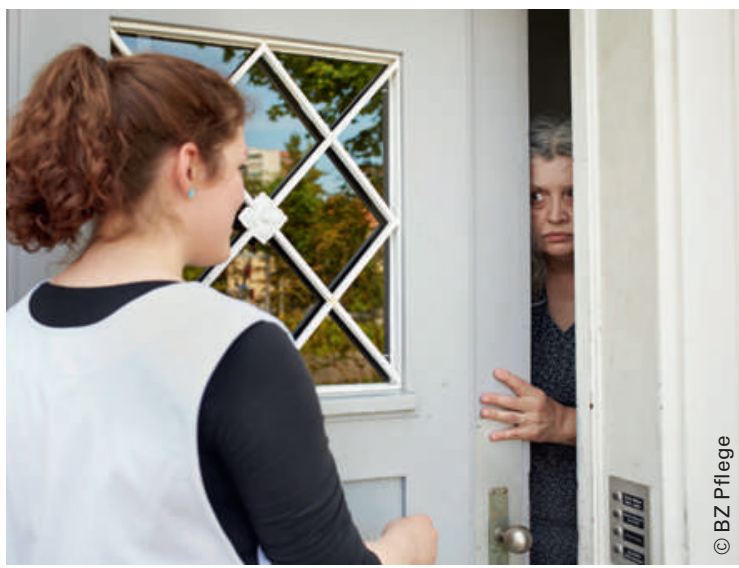

\section{Die Beziehung aufbauen}

Ärztinnen und Ärzte sowie Pflegefachpersonen haben es im Bereich Selbstvernachlässigung mit älteren Menschen zu tun, die ihre Integrität wahren wollen und Angst vor Fremdbestimmung haben. Obwohl sie mit der Komplexität des Lebens nicht mehr zurechtkommen, fällt es ihnen schwer, fremde Hilfe anzunehmen. Erika Eicher Vella, die auch als Heimärztin in einem Betagtenzentrum tätig ist, geht mit Geduld und Sorgfalt an solche Situationen heran: «Ich versuche jeweils, mich in die Situation der betroffenen Person einzufühlen.» Empathie und Geduld scheinen tatsächlich wichtige Türöffner zu sein. Sonja Bächler-Mäder, die als Co-Autorin eine neue Publikation zum Thema Selbstvernachlässigung im Alter verfasst hat (s. Box), sagt: «Der Beziehungsaufbau ist im Umgang mit betroffenen Menschen absolut zentral.» Man finde den Zugang zu diesen Personen nur über Vertrauen. «Das braucht Zeit», so Bächler-Mäder. Wichtig sei, dass man in erster Linie die Ressourcen der alten Menschen wertschätze, bevor man Interventionen vornehme.

\section{Die Perspektive wechseln}

"Auch Opposition kann eine Ressource sein», betont Gesundheits- und Pflegeexpertin Sandra Sermier, «weil damit der Wille zur Unabhängigkeit ausgedrückt wird». Fachpersonen, die den Zugang zu selbstvernachlässigten Menschen suchen, benötigen deshalb - nebst Fachkompetenz - ausgeprägte kommunikative Fähigkeiten. Mit der richtigen Gesprächstechnik, wie etwa dem aktiven Zuhören, lässt sich verhindern, dass sich im konkreten Fall Druck aufbaut und dadurch harte Fronten entstehen. Eine absolut entscheidende Rolle spielt die Haltung: «Respekt ist gefragt», betont Hausärztin Erika Eicher Vella. «Im Alter gilt es ganz besonders, die Persönlichkeit zu respektieren.» Die «Spitex RegionKöniz» setzt auf eine kontinuierliche Weiterbildung der Mitarbeitenden und stellt so eine ausrei- chende Qualifikation sicher. Präsidentin Annegret Höschele, die hauptberuflich am Berner Bildungszentrum Pflege tätig ist, erklärt: «Jede Ausbildung löst eine Auseinandersetzung mit sich selbst aus und bringt eine Persönlichkeitsentwicklung in Gang." Im Umgang mit selbstvernachlässigten Personen sei die Fähigkeit zur Reflexion und zum Perspektivenwechsel zentral.

\section{Augenmerk auf die Sozialmedizin}

Begegnet Ärztin Erika Eicher Vella in der Sprechstunde einer Patientin mit entsprechenden Symptomen, tastet sie sich langsam heran. "Ich schaue, dass ich die Person regelmässig sehe und plane die Termine an Randzeiten ein, weil ich dann mehr Zeit für Gespräche habe.» Wenn erforderlich, besucht sie ihre Patienten auch zu Hause. Sie versucht auf diesem Weg herauszufinden, welche Bedürfnisse die Person hat und mit welchen Mitteln sie unterstützt werden kann. Als Hausärztin sieht sie jedoch nur in einen Teilbereich hinein. Deshalb sei bei älteren Menschen mit Selbstvernachlässigungs-Syndrom eine enge interprofessionelle $\mathrm{Zu}$ sammenarbeit mit der Spitex zwingend notwendig. Im Übrigen findet Erike Eicher Vella, dass man auch in Ärztenetzwerken vermehrt über sozialmedizinische Themen und über Formen der Koordination diskutieren sollte. «Durch eine gute Vernetzung lassen sich Erfolge erzielen», so die Ärztin.

\section{Der runde Tisch}

In der Region Köniz übernimmt die Spitex im Einzelfall meist die Fallführung. In regelmässigen Abständen sitzen Fachpersonen, Angehörige und manchmal auch die betroffene Person selbst an einen Tisch. Man bespricht die aktuelle Situation und leitet, wenn nötig, weitere gesundheitsfördernde Massnahmen ein. Erika Eicher Vella schätzt es, wenn sie zu diesen Sitzungen eingeladen wird. Und sie regt die Spitex an, die Hausärztinnen und Hausärzte noch stärker in dieses interdisziplinäre Setting einzubeziehen. Spitex-Präsidentin Annegret Höschele unterstützt diese Bemühungen, denn sie weiss: «Wenn alle in die gleiche Richtung hin arbeiten, lassen sich viele Probleme lösen.» Im Falle des 85-jährigen Norbert K. hat es die Spitex mit Unterstützung der Ärzteschaft zwar geschafft, das Vertrauen des Patienten zu gewinnen. Allerdings verschlechterte sich der Zustand der offenen Wunde am Bein drastisch und ein stationärer Aufenthalt in einer Pflegeinstitution wurde notwendig. In vielen Fällen von Selbstvernachlässigung kann ein Netz von Fachleuten umfassende Unterstützung bieten, so dass ältere Menschen zu Hause bleiben und weiterhin selbstbestimmt leben können. 